\title{
Modification of the surface of superparamagnetic iron oxide nanoparticles to enable their safe application in humans
}

This article was published in the following Dove Press journal:

International Journal of Nanomedicine

8 November 2016

Number of times this article has been viewed

\author{
Cindy Strehl ${ }^{1,2}$ \\ Lionel Maurizi ${ }^{3}$ \\ Timo Gaber ${ }^{1,2}$ \\ Paula Hoff ${ }^{\prime}, 2$ \\ Thomas Broschard 4 \\ A Robin Poole ${ }^{5}$ \\ Heinrich Hofmann ${ }^{3}$ \\ Frank Buttgereit ${ }^{1,2}$ \\ 'Department of Rheumatology and \\ Clinical Immunology, Charité - \\ Universitätsmedizin Berlin, Berlin, \\ Germany; ${ }^{2}$ German Rheumatism \\ Research Centre (DRFZ), Berlin, \\ Germany; ${ }^{3}$ Powder Technology \\ Laboratory, Ecole Polytechnique \\ Federale de Lausanne (EPFL), \\ Lausanne, Switzerland; ${ }^{4}$ Non-Clinical \\ Safety, Merck Serono, Merck KGaA, \\ Darmstadt, Germany; ${ }^{5}$ Department of \\ Surgery, McGill University, Montreal \\ QC, Canada
}

Correspondence: Cindy Strehl Department of Rheumatology and Clinical Immunology, Charité University Hospital, Charitéplatz I, I0II7 Berlin, Germany

Tel +49304505 I3364

Fax $+49304505 \quad 13917$

Email cindy.strehl@charite.de

\begin{abstract}
Combined individually tailored methods for diagnosis and therapy (theragnostics) could be beneficial in destructive diseases, such as rheumatoid arthritis. Nanoparticles are promising candidates for theragnostics due to their excellent biocompatibility. Nanoparticle modifications, such as improved surface coating, are in development to meet various requirements, although safety concerns mean that modified nanoparticles require further review before their use in medical applications is permitted. We have previously demonstrated that iron oxide nanoparticles with amino-polyvinyl alcohol (a-PVA) adsorbed on their surfaces have the unwanted effect of increasing human immune cell cytokine secretion. We hypothesized that this immune response was caused by free-floating PVA. The aim of the present study was to prevent unwanted immune reactions by further surface modification of the a-PVA nanoparticles. After cross-linking of PVA to nanoparticles to produce PVA-grafted nanoparticles, and reduction of their zeta potential, the effects on cell viability and cytokine secretion were analyzed. PVAgrafted nanoparticles still stimulated elevated cytokine secretion from human immune cells; however, this was inhibited after reduction of the zeta potential. In conclusion, covalent crosslinking of PVA to nanoparticles and adjustment of the surface charge rendered them nontoxic to immune cells, nonimmunogenic, and potentially suitable for use as theragnostic agents.
\end{abstract}

Keywords: iron oxide nanoparticles, cytokine secretion, cell viability, polyvinyl alcohol, cross-linking, surface charge

\section{Introduction}

The rising costs of human health care clearly reflect an urgent demand for improved medical care. Treatment strategies include both early detection of disease, ideally before damage can occur, and targeting of therapeutic measures, thereby diminishing unwanted adverse effects. A combination of both individually tailored diagnosis and therapy, the so called "theragnostic approach", would be desirable. Nanoparticles (NP) are very promising tools in this field, and are already used in various medical applications. ${ }^{1-5}$

Organic NP include liposomes, dendrimer constructs, ALB-bound formulations, and polymeric NP, in which the specific, potentially therapeutic molecules are enclosed. These organic NP are mainly used for drug delivery, because they display improved pharmacokinetics and pharmacodynamics compared to the drugs themselves. Furthermore, the organic NP delivery system is biocompatible and biodegradable. ${ }^{6}$ This is not the case for inorganic NP, such as metallic NP, quantum dots, or carbon nanotubes, which are mainly used for diagnostic purposes - for example, as contrast enhancers for magnetic resonance imaging (MRI). ${ }^{7-11}$ The therapeutic application of inorganic NP is constantly developing, although to a lesser extent than that of organic NP. ${ }^{6}$ Examples 
of therapeutic applications are drug delivery systems, ${ }^{12,13}$ stem cell tracking, ${ }^{14,15}$ and thermotherapy. ${ }^{3,16}$

Superparamagnetic iron oxide nanoparticles (SPION) are promising inorganic NP. They have been intensively investigated over the last 20 years for various in vitro and in vivo biomedical applications. ${ }^{1}$ One advantage they offer is that they have excellent physicochemical properties, leading to improved efficacy and specificity; for example, as contrast enhancers in in vivo imaging techniques. They exhibit high magnetization in the presence of an external magnetic field, whereas this magnetization is lost when the external magnetic field is removed. ${ }^{17}$ MRI is an important noninvasive tool to visualize organs, tissues, and pathological changes or treatment outcomes. ${ }^{18,19}$ In the field of chronic, systemic inflammatory autoimmune diseases, such as rheumatoid arthritis, there is a need for close and regular monitoring of disease activity. Achieving this goal is important, since damage to cartilage and bone as a consequence of this destructive disease is irreversible; ${ }^{20}$ hence, early diagnosis is needed. This may be facilitated by use of specially developed SPION as effective, specific, and safe theragnostic agents.

The surface of NP can be coated with biocompatible substances to ensure that their application is safe. One example is the siloxane-coated SPION, ferumoxsil (trade name Lumirem ${ }^{\circledR}$ or GastroMARK ${ }^{\circledR}$ ), which is already used as a contrast agent in gastroenterology, to distinguish the bowel from other abdominal structures. ${ }^{21}$

Furthermore, SPION are endocytosed by cells, particularly immune cells such as monocytes and macrophages, which are dedicated phagocytes that migrate into inflamed tissues. ${ }^{22-25}$ SPION can be further functionalized by surface coating using proteins, peptides, antibodies, polymers, carbohydrates, aptamers, DNA, RNA, or oligosaccharides. ${ }^{17}$ The composition and characteristics of the SPION surface have a strong influence on their stability, distribution, and biocompatibility, with regard to cellular uptake and cytotoxicity. Although commercially available SPION have been approved for human use, they present some risks when used for treatment purposes, and these are currently being reevaluated. ${ }^{4,26-29}$ Currently, there are no accepted standardized protocols or toxicity assays available that could help to compare the properties of the many SPION which are under development. Moreover, there is also a lack of standardized preclinical and clinical study protocols for this purpose.

Nevertheless, in vitro assays demonstrate that polyvinyl alcohol (PVA) coated SPION can alter the metabolism of immune cells. Previously, we showed that amino (a)-PVASPION do not affect the survival ${ }^{25}$ or activity ${ }^{30}$ of human immune cells; however, these particles do cause unwanted immune reactions, in terms of increased cytokine secretion. ${ }^{25}$
To enable safe medical application of these NP, modification of a-PVA-SPION is necessary. Because the coating of these NP was not optimally controlled in former experiments, it was not clear if the effects observed were due to the coated SPION themselves or to free-floating PVA polymer, which could trigger an immune reaction.

In this study, we demonstrate that it is advantageous to precisely modify the SPION surface and show how this positively influences the responses of human immune cells.

\section{Materials and methods Materials}

All chemicals were of analytical reagent grade and used without further purification. Three types of PVA were used during this study. PVA Mowiol 4-88 $\left(\mathrm{PVA}_{4-88}\right)$ was provided by Merck ${ }^{\circledR} \mathrm{KGaA}$ (Darmstadt, Germany). PVA $_{488}$ is a classical PVA without any functional group, has a molecular weight of 30,000 , and a degree of hydrolysis of $88 \%$. Amino-functionalized PVA M12 $\left(\mathrm{PVA}_{\mathrm{M} 12}\right)$, also used in a previous study, ${ }^{22}$ was supplied by Erkol $^{\circledR}$ (Tarragona, Spain). PVA $_{\mathrm{M} 12}$ contains $4 \%$ amino $\left(\mathrm{NH}_{2}\right)$ groups and has a molecular weight of between 80,000 and 120,000. PVA KL506 (PVA ${ }_{\text {KL } 506}$ ) was supplied by Kuraray ${ }^{\circledR}$ (Kuraray Europe GmbH, Hattersheim am Main, Germany). $\mathrm{PVA}_{\mathrm{KL} 506}$ has a random distribution of carboxyl groups $(\mathrm{COOH})$ and a molecular weight of between 30,000 and 50,000.

\section{SPION synthesis and characterization} Preparation of naked SPION and PVA-coated SPION Naked SPION were synthesized according to a published protocol. ${ }^{15}$ a-PVAcoated SPION (a-PVA-SPION), were prepared as described previously, using a mixture of $\mathrm{PVA}_{488}$ and $\mathrm{PVA}_{\mathrm{M} 12}$ at a mass ratio of $45 / 1$ respectively. ${ }^{22}$ The $\mathrm{PVA}_{488}$ solution was prepared by dissolving this polymer in water at $10 \%$ mass/ volume. All solutions were heated for 30 minutes at $90^{\circ} \mathrm{C}$ to improve polymer solubility. The polymer solution was then passed through a sterile filter (pore size, $200 \mathrm{~nm}$ ). Ultrapure deionized water (DI water from Seralpur delta; Seral GmbH, Ransbach-Baumbach, Germany) was used throughout the synthesis. To obtain PVA-grafted SPION, a preliminary step of PVA coating was performed with a chemical mass ratio of PVA to iron (PVA/Fe) of 7 ( PVA $_{4-88}$-SPION-R7) by mixing 10 volumes of naked SPION with 7 volumes of PVA $_{488}$ solution and 3 volumes of DI water. The preliminary $\mathrm{PVA}_{488}{ }_{-} \mathrm{SPION}-\mathrm{R} 7$ were stored for 1 week at $4^{\circ} \mathrm{C}$ before synthesis was continued.

\section{Grafting (covalent linking) of PVA onto SPION using} a silica cross-linking agent: a-PVA-grafted-SPION

PVA was grafted onto the surface of SPION using a silica precursor [APTES: (3-aminopropyl)triethoxysilane] and 
PVA $_{4-88}$-SPION-R7. As described previously, ${ }^{31}$ this method allows reproducible synthesis of PVA-grafted SPION by cross-linking the polymer onto the surface of SPION with silica. Furthermore, APTES provides amino groups on the surface of $\mathrm{PVA}_{4-88}$-SPION-R7 to have identical surface as previously used a-PVA-SPION functionalized with $\mathrm{PVA}_{\mathrm{M} 12}{ }^{22}$ For one batch of synthesis, $25 \mathrm{~mL}$ of $\mathrm{PVA}_{4-88}$ SPION-R7 was diluted in $900 \mathrm{~mL}$ of DI water. The $\mathrm{pH}$ was adjusted to $\mathrm{pH} 10$ with ammonia (25\%) and the suspension heated to $60^{\circ} \mathrm{C}$. Then, $10 \mathrm{~mL}$ of APTES was added and the reaction continued for 5 hours. The suspension was neutralized to $\mathrm{pH} 7-8$ using $\mathrm{HCl}(6 \mathrm{M})$ before loading $25 \mathrm{~mL}$ into a magnetic separator filled with thin stainless steel wires (Frantz ${ }^{\circledR}$ Canister Separator, Model L-1CN; S. G. Frantz Company Inc., Tullytown, PA, USA), using a peristaltic pump set at $14 \mathrm{~mL} / \mathrm{min}$ and a magnetic field set at 1 tesla $(120 \mathrm{~V}, 1.8 \mathrm{~A})$ (Figure S1). After loading (approximately 1 hour), the reactor was rinsed with an equivalent of 10 reactor volumes $(10 \times 25 \mathrm{~mL})$ of DI water at $14 \mathrm{~mL} / \mathrm{min}$ for 20 minutes. The magnetic field was then removed and the suspension released from the separator. The suspension obtained was concentrated magnetically to obtain a-PVAgrafted-SPION at $5 \mathrm{mg}$ of Fe per $\mathrm{mL}$.

For some experiments, the charge of a-PVA-graftedSPION was adjusted using a very small amount of PVA ${ }_{\mathrm{KL} 506}$ in a mass ratio of $\mathrm{PVA}_{\mathrm{KL} 506} / \mathrm{Fe}$ of 0.05 . The two different types of NP generated were a-PVA-grafted-SPION $(+)$ (with decreased charge) and a-PVA-grafted-SPION $(++)$ (without decreased charge).

\section{Characterization of NP}

The mean diameter of the magnetic cores $\left(\mathrm{d}_{\text {core }}, \mathrm{nm}\right)$ was determined by counting 400 diameters/fields using a transmission electron microscope (CM12; Philips Electron Optics, Zürich, Switzerland) at 45,000× magnification. Hydrodynamic diameter $\left(\mathrm{d}_{\mathrm{H}}, \mathrm{nm}\right)$ and zeta potential ( $\mathrm{Z}$ potential, $\mathrm{mV}$ ) were measured at $\mathrm{pH} 7$, at an ionic strength of $10 \mathrm{mM} \mathrm{NaCl}$, by dynamic light scattering, using a Malvern NanoZS (Nanosizer 7.2 software; Malvern Instruments, Malvern, UK) and a theoretical refractive index of magnetite of $2.42 .{ }^{32}$ The saturation magnetization $(\mathrm{emu} / \mathrm{g})$ was obtained from lyophilized SPION before PVA coating, using a superconducting quantum interference device. ${ }^{33}$ The chemical composition of SPION, (ie, the concentration of iron in $\mathrm{mg}$ of $\mathrm{Fe}$ per $\mathrm{mL}$ ), was determined using magnetic susceptibility methods. ${ }^{34}$ In addition, the amount of PVA (PVA/Fe in mgPVA $/ \mathrm{mgFe}$ ) present on the surface of SPION was calculated by thermogravimetric analyses (TGA; Mettler Toledo TGA/SDTA 851e [Columbus, Ohio, USA]) of the loss of mass that occurred upon heating from $150^{\circ} \mathrm{C}$ to $800^{\circ} \mathrm{C}\left(10^{\circ} \mathrm{C} / \mathrm{min}\right)$ under air $(30 \mathrm{~mL} / \mathrm{min})$. This provided the PVA/Fe.

\section{Antibodies and reagents}

Privigen ${ }^{\circledR}$ human immunoglobulin from CSL Behring (King of Prussia, PA, USA) was used for flow cytometry. Anti-human-CD14-APC-Cy7 and anti-human-CD19-PECy7 were from BioLegend (San Diego, CA, USA), antihuman-CD3-Pacific-blue and Annexin V/7AAD apoptosis kits were from BD Biosciences (San Jose, CA, USA), and anti-human-CD15-APC was from Miltenyi Biotech $\mathrm{GmbH}$ (Bergisch Gladbach, Germany). Lectin from Phaseolus vulgaris (PHA-L) and lipopolysaccharide (LPS) from SigmaAldrich Co. (St Louis, MO, USA) were used for immune cell stimulation. The commercially available MRI contrast agent ferumoxsil (Lumirem ${ }^{\circledR}$; Guerbet, Villepinte, France) was used as a negative control for the final comparative analysis of different NP preparations.

\section{Whole blood cell viability analysis: sample preparation and stimulation}

Venous blood obtained from healthy donors was collected in heparinized tubes. Healthy donors provided written informed consent before enrollment in the study. The study protocol was approved by the responsible local administrative body and ethics committee of Charité University Medicine Berlin in accordance with the Declaration of Helsinki.

For each condition, $100 \mu \mathrm{L}$ of fresh whole blood samples were diluted with $100 \mu \mathrm{L}$ of Roswell Park Memorial Institute (RPMI) 1640 culture medium (GIBCO; Thermo Fisher Scientific, Waltham, MA, USA) supplemented with $100 \mathrm{U} / \mathrm{mL}$ penicillin $\mathrm{G}, 100 \mu \mathrm{g} / \mathrm{mL}$ streptomycin (both from PAA Laboratories $\mathrm{GmbH}$, Pasching, Austria), and $50 \mu \mathrm{M}$ $\beta$-mercaptoethanol (Sigma-Aldrich Co.) in MegaBlock ${ }^{\circledR}$ 96-well $1.2 \mathrm{~mL}$ deep-well plates (Sarstedt AG \& Co., Nuembrecht, Germany).

Cells were stimulated with LPS $(1 \mu \mathrm{g} / \mathrm{mL})$, PHA-L (5 $\mu \mathrm{g} / \mathrm{mL})$, a-PVA-SPION (1, 10 and, $100 \mu \mathrm{g} / \mathrm{mL})$, or a-PVA $(1,10$ and, $100 \mu \mathrm{g} / \mathrm{mL})$, or were untreated, and were incubated for 20 hours in a humidified incubator at $37^{\circ} \mathrm{C}$ $\left(18 \% \mathrm{O}_{2} / 5 \% \mathrm{CO}_{2}\right)$. Afterwards, supernatants were collected, immediately frozen, and stored at $-80^{\circ} \mathrm{C}$ for cytokine secretion analysis, and cells were prepared for flow cytometry (see below).

\section{Quantification of secreted cytokines}

Secreted cytokines were quantified using a Bio-Plex ${ }^{\circledR}$ Pro Cytokine 27-Plex Panel Human Group I on a Bio-Plex ${ }^{\mathbb{B}}$ 
200 system with high-throughput fluidics (all from Bio-Rad Laboratories Inc., Hercules, CA, USA).

\section{Analysis of cell survival by flow cytometry}

Erythrocytes were lysed by adding $900 \mu \mathrm{L}$ of erythrocyte lysis buffer (0.01 $\mathrm{M} \mathrm{KHCO}_{3}, \mathrm{pH} 7.5 ; 0.155 \mathrm{M} \mathrm{NH}_{4} \mathrm{Cl}$; $0.1 \mathrm{mM}$ ethylenediaminetetraacetic acid) to $200 \mu \mathrm{L}$ whole blood samples. After incubation for 10 minutes at room temperature, white blood cells were collected by centrifugation and the procedure was repeated once. Cell pellets were then washed with phosphate-buffered saline (PBS) and nonspecific binding was blocked by preincubation with human immunoglobulin $(5 \mathrm{mg} / \mathrm{mL})$ for 10 minutes at $4{ }^{\circ} \mathrm{C} .{ }^{25}$ After washing the cells with PBS, an antibody-mix (anti-CD3, anti-CD14, anti-CD19, and anti-CD15; all antibodies diluted 1/50 in PBS) was added, mixed, and the samples were incubated for 15 minutes at $4^{\circ} \mathrm{C}$ in the dark. Cells were washed twice and Annexin V-PE/7-AAD staining was performed according to the manufacturer's instructions. Cells were analyzed within 1 hour by flow cytometry using a MACSQuant ${ }^{\circledR}$ analyzer equipped with a MACS $^{\circledR}$ MiniSampler (Miltenyi Biotech $\mathrm{GmbH})$. The acquired data were analyzed using FlowJo 7.6.1 software (Tree Star Inc, Ashland, OR, USA).

\section{Statistical analyses}

Flow cytometry data were analyzed using GraphPad Prism Software (GraphPad Software, Inc., La Jolla, CA, USA) and are presented as box and whisker plots (whiskers from minimum to maximum), or mean with standard deviation, of at least eight independent experiments. Multiple comparisons were analyzed by one- or two-way analysis of variance as indicated, with Bonferroni's multiple comparison post hoc tests for normally distributed data. Probability values of $P<0.05$ were considered to be statistically significant.

\section{Results}

\section{SPION synthesis and characterization}

The method used to obtain a-PVA-grafted-SPION allowed the production of $50 \mathrm{~mL}$ of $\mathrm{NP}$ (at $5 \mathrm{mg}$ of Fe per $\mathrm{mL}$ ) per day in a reproducible manner, with a yield of approximately $80 \%$. The three types of NP used in this study [PVA-SPION, a-PVA-grafted-SPION (++), and a-PVA-grafted-SPION (+)] had comparable hydrodynamic diameters and $\mathrm{Z}$ potentials. The average hydrodynamic diameter of a-PVA-SPION, a-PVA-grafted-SPION (++), and a-PVA-grafted-SPION (+) were $31 \pm 10,28 \pm 8$, and $32 \pm 7 \mathrm{~nm}$, respectively. The three functionalized SPION have higher hydrodynamic diameter than naked SPION (14 $\pm 2 \mathrm{~nm})$ because of the addition of polymers, which increase their hydrodynamic diameters. The hydrodynamic diameters of the synthesized SPION are approximately ten times smaller than that of ferumoxsil, which is approximately $300 \mathrm{~nm} .{ }^{35}$ The PVA coating and grafting also improved the stability of the naked SPION in either biological buffer or cell medium (RPMI from GIBCO in this study) for at least 2 months (observed via dynamic light scattering measurements over time). The PVA surface functionalization did neither affect core sizes (all $\mathrm{d}_{\text {cores }}$ were $7.2 \pm 2.5 \mathrm{~nm}$ ), nor the saturation magnetization $(54 \mathrm{emu} / \mathrm{g})$. The $\mathrm{d}_{\text {cores }}$ and saturation magnetization of the synthesized SPION have very similar values to those of ferumoxsil, which are $8.4 \pm 2.5 \mathrm{~nm}$ and 49.4 $\pm 0.1 \mathrm{emu} / \mathrm{g}$, respectively. ${ }^{35}$ The APTES used for the grafting procedure closely resembles the chemistry used to stabilize ferumoxsil, which also uses silica (siloxane coating). ${ }^{36}$ The PVA coating could not be observed by transmission electron microscope, due to the low contrast of the polymer (Figure S2); however, the lack of influence of PVA on the size and morphology of the magnetic cores was evident.

The $\mathrm{Z}$ potentials of the three functionalized PVA-SPION were all positive at $\mathrm{pH} 7$, at $+22 \pm 6,+31 \pm 5$, and $+21 \pm 2 \mathrm{mV}$ for a-PVA-SPION, a-PVA-grafted-SPION (++), and a-PVAgrafted-SPION $(+)$, respectively. In all three cases, the chemical groups providing a positive charge at the surface were identical, namely amino groups $\left(\mathrm{NH}_{3}{ }^{+} / \mathrm{NH}_{2}\right)$, resulting from either PVA $_{\mathrm{M} 12}$ used in the coating process for a-PVA-SPION or APTES for a-PVA-grafted-SPION.

The main difference between the a-PVA-SPION with adsorbed polymer and the a-PVA-grafted-SPION (Table 1) was a considerable reduction in the amount of PVA used in the protocol to produce grafted NP. Chemical analyses and

Table I Physicochemical characterization of SPION with adsorbed amino-PVA (a-PVA-SPION) and grafted PVA with amino groups (a-PVA-grafted-SPION)

\begin{tabular}{|c|c|c|c|c|c|c|}
\hline Nanoparticles & $d_{\text {core }}(\mathrm{nm})$ & $d_{H}(n m)$ & $\mathbf{Z}$ potential $(\mathrm{mV})$ & Sat mag (emu/g) & Conc $\left(\mathrm{mg}_{\mathrm{Fe}} / \mathrm{mL}\right)$ & PVA/Fe $\mathrm{mg}_{\mathrm{PVA}} / \mathrm{mg}_{\mathrm{Fe}}$ \\
\hline Naked SPION & $7.2 \pm 2.5$ & $14 \pm 2$ & $1 \pm 3$ & 54 & 10 & 0 \\
\hline a-PVA-SPION & $7.2 \pm 2.5$ & $31 \pm 10$ & $22 \pm 6$ & 54 & 5 & 9 \\
\hline a-PVA-grafted-SPION $(++)$ & $7.2 \pm 2.5$ & $28 \pm 8$ & $3 I \pm 5$ & 54 & 5 & 1 \\
\hline a-PVA-grafted-SPION (+) & $7.2 \pm 2.5$ & $32 \pm 7$ & $2 I \pm 2$ & 54 & 5 & I \\
\hline
\end{tabular}

Abbreviations: $\mathrm{d}_{\text {core }}$, mean diameter of the magnetic core; $\mathrm{d}_{\mathrm{H}}$, hydrodynamic diameter of a-PVA-SPION; $Z$ potential, zeta potential; sat mag, saturation magnetization; conc, concentration of SPION; PVA/Fe, chemical mass ratio of PVA to iron; SPION, superparamagnetic iron oxide nanoparticles; PVA, polyvinyl alcohol. 
the PVA/Fe mass ratios clearly demonstrated that the quantity of PVA used to generate the a-PVA-grafted-SPION with the grafting method is much lower (PVA/Fe mass ratio of 1) than that used to produce a-PVA-SPION by the adsorption method (PVA/Fe mass ratio of 9). These analyses show that, compared with passive surface adsorption, covalent grafting of PVA requires up to $90 \%$ less polymer to stabilize the SPION.

A PVA/Fe ratio of 1 is in accordance with our previous work which showed that the minimal ratio needed to stabilize SPION is between 1 and 2. ${ }^{31,37}$ Furthermore, it also demonstrates that the PVA adsorbed on a-PVA-SPION is free-floating and would be detached from the surface when diluted for biological experimentation. A summary of the proposed mechanism of surface functionalization of SPION with PVA is shown in Figure 1. Finally, no difference was observed between the amounts of PVA on $(+)$ and (++) a-PVA-grafted-SPION, demonstrating that only a small amount of PVA is required to change the $\mathrm{Z}$ potential of these particles.

Importantly, the grafting method described here enables the creation of a stable a-PVA-SPION without any freefloating polymer, but with physicochemical properties comparable to adsorbed-PVA-coated SPION.

\section{Effects of free PVA on ILI $\beta$ secretion in human immune cells}

Previously, we found thata-PVA-SPION significantly increased IL1 $\beta$ secretion, in a dose-dependent manner; ${ }^{25}$ therefore,

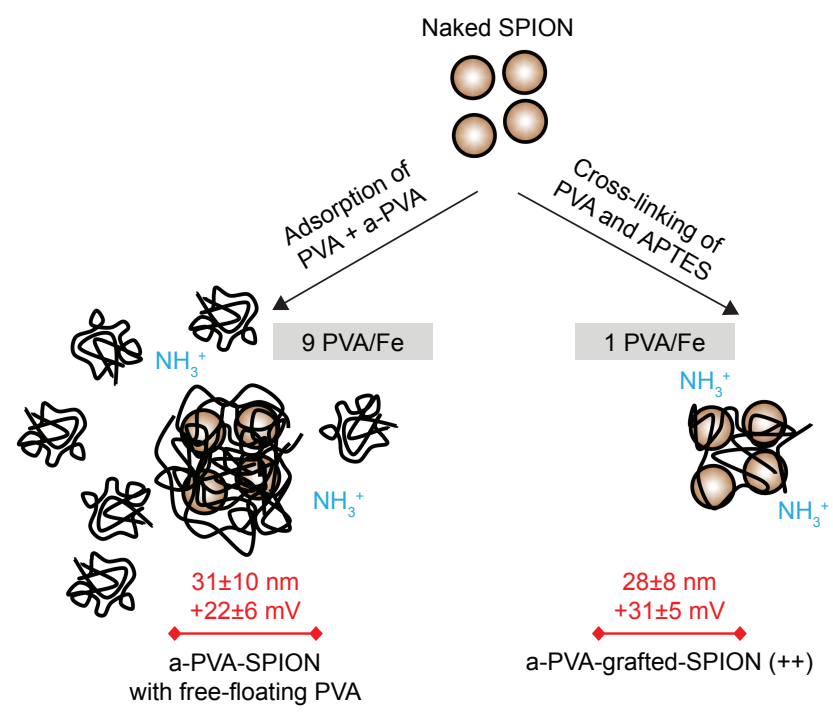

Figure I Schematic representation of the different methods used to functionalize naked SPION (brown circles) with PVA (black lines).

Notes: The silica precursor (APTES) grafts the PVA onto naked SPION and provides amino groups and positive charges to obtain a-PVA-grafted-SPION comparable in size, chemistry, and charge to a-PVA-SPION (with adsorbed PVA).

Abbreviations: SPION, superparamagnetic iron oxide nanoparticles; a-PVA, aminopolyvinyl alcohol; PVA, polyvinyl alcohol; AAPTES, (3-aminopropyl)triethoxysilane. we investigated whether this effect could result from free a-PVA present in the a-PVA-SPION preparation. Whole blood samples obtained from eight healthy donors were incubated with varying concentrations of a-PVA, and IL1 $\beta$ secretion was analyzed in supernatants. Unstimulated cells secreted IL1 $\beta$ at a median concentration of $15.5 \mathrm{pg} / \mathrm{mL}$, whereas incubation with LPS increased the IL1 $\beta$ secretion to $529.1 \mathrm{pg} / \mathrm{mL}$. Incubation with a-PVA alone for 20 hours led to an increase relative to unstimulated secretion levels in whole blood samples, with median values of IL1 $\beta$ of $62.45,371.2$, and $653.5 \mathrm{pg} / \mathrm{mL}$ at 1,10 , and $100 \mu \mathrm{g} / \mathrm{mL}$, respectively (Figure 2A). Thus, the effect of a-PVA-SPION on IL1 $\beta$ secretion $^{25}$ may be triggered by free a-PVA.

\section{Effects of modified a-PVA-grafted-SPION on human immune cells}

Next, the effects of a-PVA-grafted-SPION (++) on immune cell survival and cytokine secretion were analyzed. As already reported for a-PVA-SPION,${ }^{25}$ we found no significant influence of a-PVA-grafted-SPION (++) on apoptosis or viability of human immune cells at concentrations up to $100 \mu \mathrm{g} / \mathrm{mL}$ (data not shown). Secreted cytokines were analyzed in the supernatants collected from these whole blood viability experiments $(\mathrm{n}=9)$ using the Bio-Plex ${ }^{\circledR}$ Suspension array system. We found significantly increased secretion of the following cytokines in the presence of a-PVA-graftedSPION (++) (at $100 \mu \mathrm{g} / \mathrm{mL})$ compared with the untreated control: IL6, IL8, IL10, IL12, IL17, MCP-1, MIP1a, MIP1b, FGF-basic, VEGF, and GM-CSF (Figure 2B). These effects differed from those observed with a-PVASPION $;{ }^{25}$ however, cytokine secretion did increase. Prior quantitative analyses have demonstrated that the size and shape of the charge of NP are the key factors influencing $\mathrm{NP}-$ cell interactions, especially their intracellular accumulation..$^{38}$ The SPION used in the present study do not differ in their type of surface coating; however, the process of PVA cross-linking to the SPION led to the a-PVA-grafted-SPION carrying a slightly higher positive charge, in comparison with a-PVA-SPION. Therefore, we reduced the $\mathrm{Z}$ potential of a-PVA-grafted-SPION (++) further, to produce a-PVAgrafted-SPION $(+)$ and analyzed the effects of this preparation on human immune cells.

We then compared the effects of the three NP preparations [a-PVA-SPION, a-PVA-grafted-SPION (++), and a-PVA-grafted-SPION (+)] and ferumoxsil, which was used at concentrations of 1 and $10 \mu \mathrm{g} / \mathrm{mL}$ because higher concentrations were not feasible due to the low concentration of the commercially available suspension. Ferumoxsil is a suspension of siloxane-coated superparamagnetic iron 

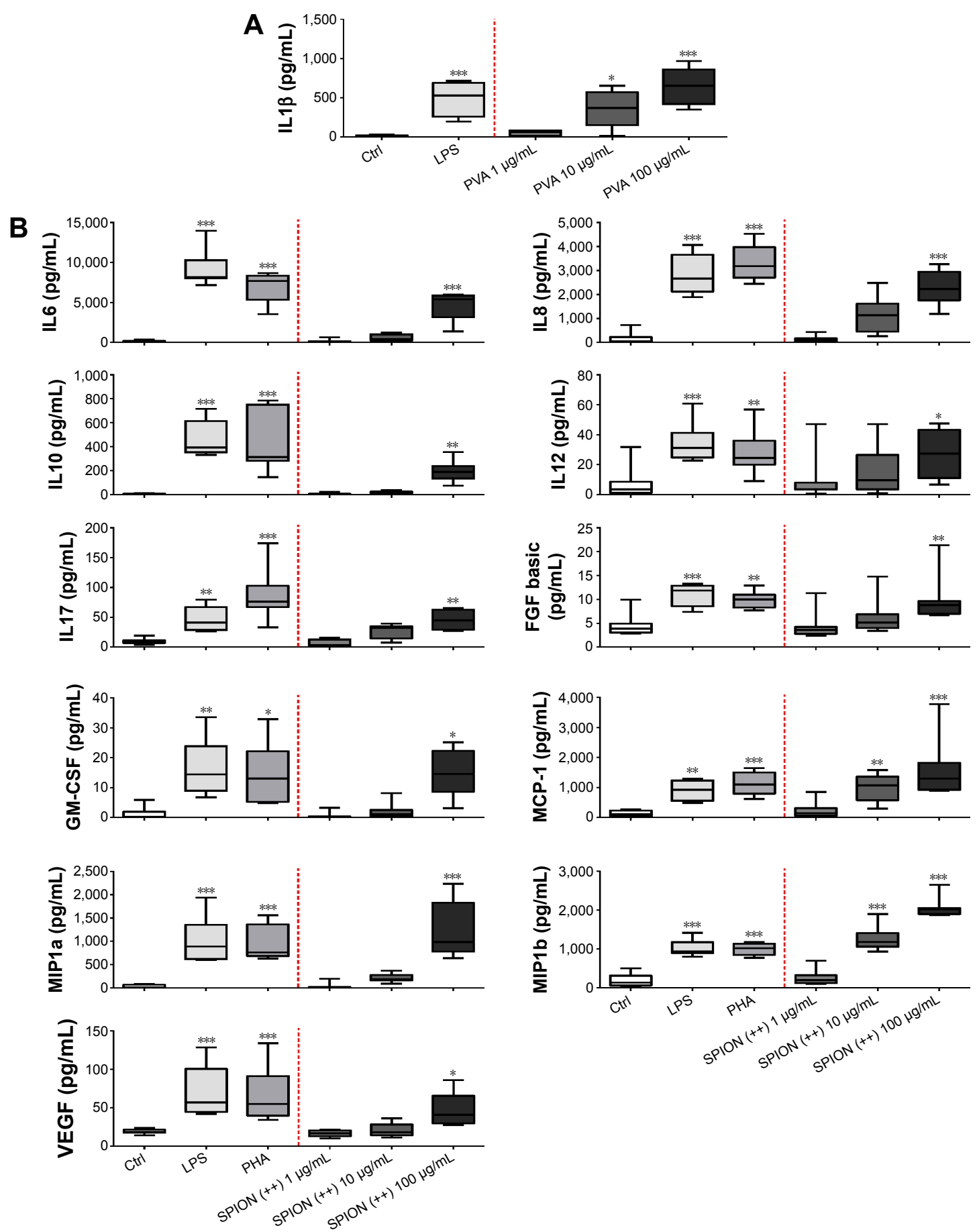

Figure 2 Influence of free-floating PVA and a-PVA-grafted-SPION $(++)$ on whole blood cytokine secretion in human immune cells.

Notes: (A) Free-floating PVA. ILI $\beta$ secretion was analyzed in supernatants collected from human whole blood samples obtained from healthy donors ( $\mathrm{n}=8$ ) after 20 hours incubation with PVA. (B) a-PVA-grafted-SPION (++). Supernatants were collected from whole blood assays [blood samples obtained from healthy donors ( $\mathrm{n}=9$ )] and analyzed for secreted cytokines. Data are presented as box and whisker plots; whiskers represent minimum to maximum. One-way ANOVA: $* * * P<0.00 \mathrm{I} ; * * P<0.0 \mathrm{I} ; * \mathrm{P}<0.05$. Abbreviations: SPION, superparamagnetic iron oxide nanoparticles; a-PVA, amino-polyvinyl alcohol; ANOVA, analysis of variance; ctrl, control; LPS, lipopolysaccharide; PVA, polyvinyl alcohol; LPS, lipopolysaccharide.

oxide crystals, and is in current routine use as a diagnostic contrast agent for MRI examinations of the stomach and bowels.

To analyze the influence of the different NP preparations on human immune cells, we quantified the number of
Annexin $\mathrm{V}$ positive (apoptotic) or 7AAD positive (dead) cells, in addition to cell-specific extracellular markers (CDantigens) in whole blood samples obtained from ten healthy donors incubated with NP. We did not find any significant effect of any of the different NP preparations on apoptosis 
and viability in the cell types analyzed (Figure 3A). Furthermore, the numbers of apoptotic or dead cells were very similar to those identified in cells incubated with ferumoxsil.

Analysis of the induction of cytokine secretion triggered by incubation with different concentrations of NP clearly demonstrated that the effects of a-PVA-grafted-SPION $(+)$ are lower compared with those of the other formulations. There was a significantly increased secretion of the following cytokines after incubation of cells with NP at $100 \mu \mathrm{g} / \mathrm{mL}$, compared with the untreated control (Table S1; Figure 3B): a-PVA-SPION led to an increase in IL1 $\beta$, IL4, IL6, IL8, G-CSF, IL17, MCP-1, MIP1a, MIP1b, PDGF, and TNF $\alpha$; and a-PVA-grafted-SPION (++) led to an increase in IL6, IL8, IL17, G-CSF, MCP-1, MIP1a, MIP1b, and TNFo; whereas a-PVA-grafted-SPION (+) only stimulated the production of MCP-1 and MIP1b. All other cytokines were unaffected. Ferumoxsil treatment did not cause elevation of the concentration of any cytokine (Figure 3B; all median concentrations are listed in Table S1).

A representative example, demonstrating the results of analysis of IL17 secretion from whole blood samples, is shown in Figure 3C. Unstimulated cells secrete IL17 at a median concentration of $11 \mathrm{pg} / \mathrm{mL}$, whereas incubation with LPS or PHA-L increased IL17 secretion to 72 and $76.6 \mathrm{pg} / \mathrm{mL}$, respectively. Incubation with a-PVA-SPION at 1,10 , and $100 \mu \mathrm{g} / \mathrm{mL}$ led to median values of IL17 of 11 , 49.9, and $66.1 \mathrm{pg} / \mathrm{mL}$ secreted from whole blood samples, respectively. Incubation with a-PVA-grafted-SPION (++) led to a slight decrease in IL17 secretion compared with
A

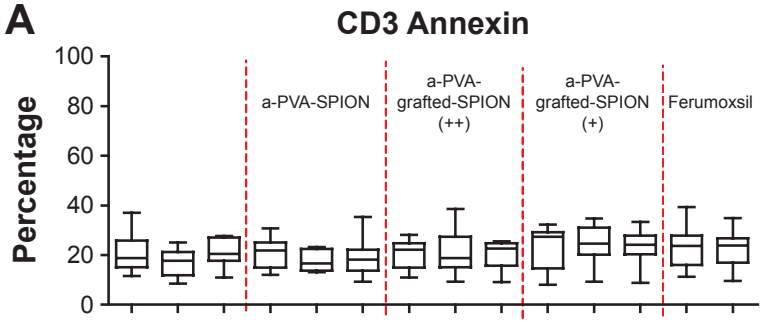

CD14 Annexin

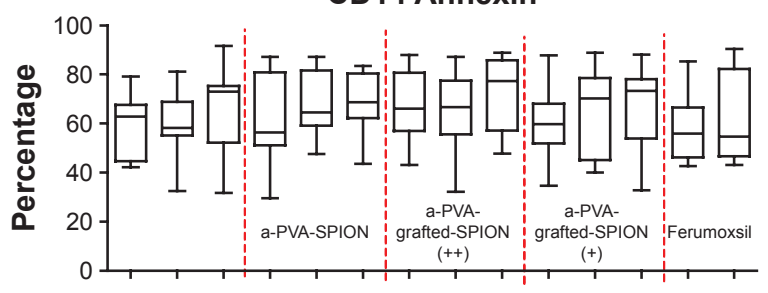

CD15 Annexin

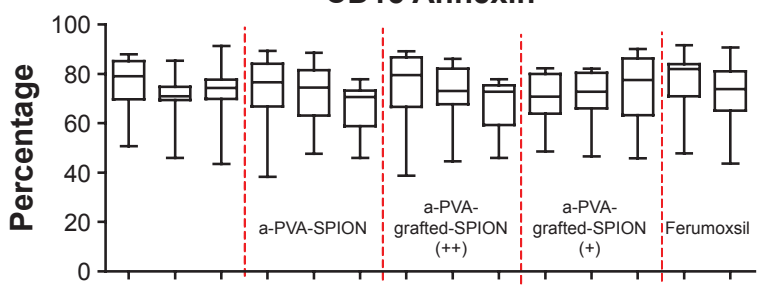

CD19 Annexin

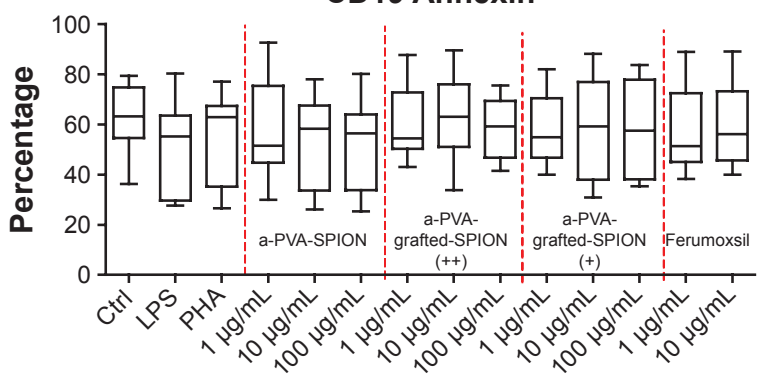

CD3 7AAD

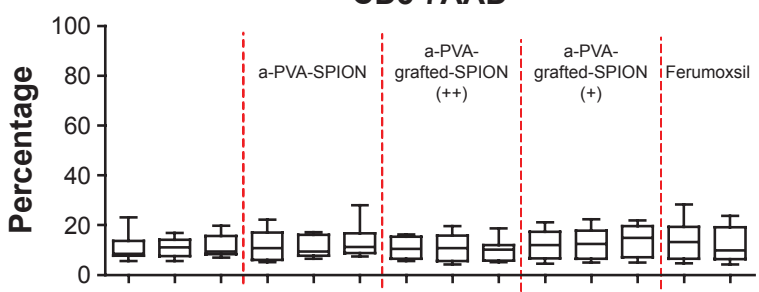

CD14 7AAD

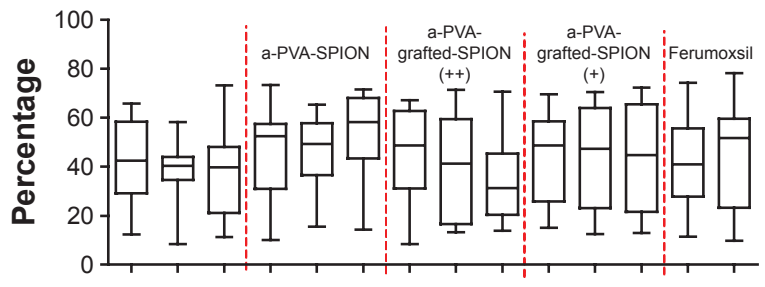

CD15 7AAD
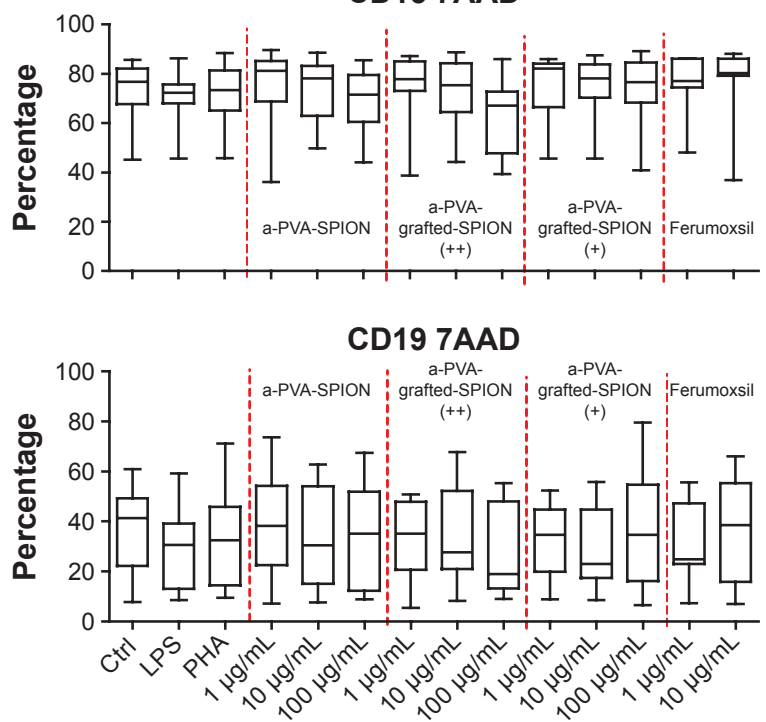

Figure 3 (Continued) 


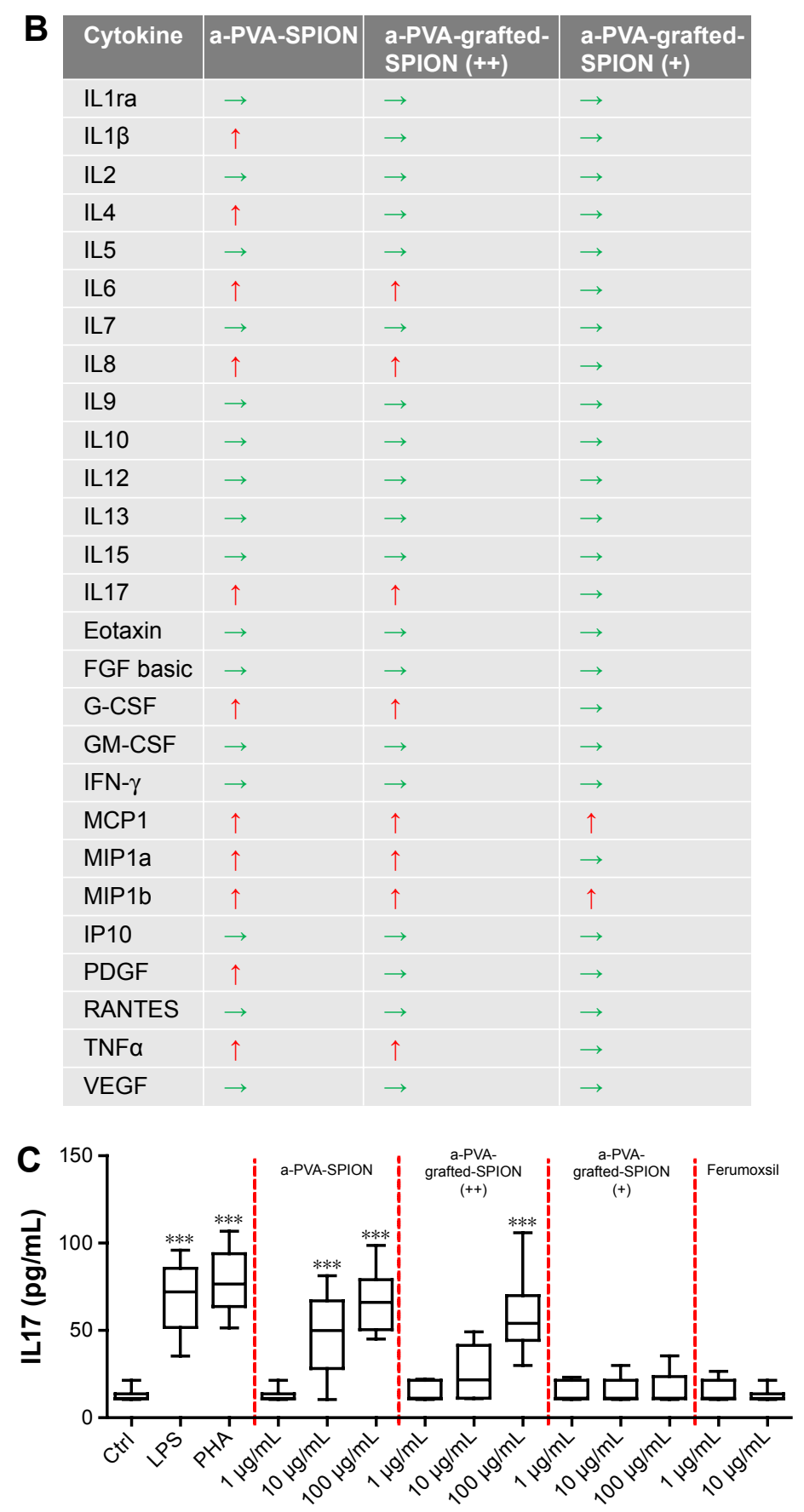

Figure 3 Influence of different preparations of NP on the viability and cytokine secretion of human immune cells.

Notes: (A) Whole blood cell survival analysis was performed with blood samples obtained from healthy donors ( $\mathrm{n}=10$ ). Cells were analyzed with Annexin $V$ (an apoptosis marker) and 7AAD (a necrosis marker). (B) Supernatants were collected from whole cell blood survival assays and analyzed for secreted cytokines. Red arrow, cytokines that were significantly increased compared to untreated controls at a SPION concentration of $100 \mu g / \mathrm{mL}$; green arrow, cytokines that remained unchanged. One example, ILI7 secretion, is presented in detail in (C). Data are presented as box and whisker plots; whiskers represent minimum to maximum. One-way ANOVA: ***P $<0.00$ I.

Abbreviations: NP, nanoparticles; SPION, superparamagnetic iron oxide nanoparticles; ANOVA, analysis of variance; a-PVA, amino-polyvinyl alcohol; ctrl, control; LPS, lipopolysaccharide; PVA, polyvinyl alcohol.

a-PVA-SPION, with median values of $11,21.7$, and 54.1 $\mathrm{pg} / \mathrm{mL}$ at 1,10 , and $100 \mu \mathrm{g} / \mathrm{mL}$, respectively. Interestingly, incubation with a-PVA-grafted-SPION (+) did not lead to an increase in the secretion of IL17, even at $100 \mu \mathrm{g} / \mathrm{mL}$. Finally, ferumoxsil had no effect on IL17 secretion (median values $11 \mathrm{pg} / \mathrm{mL}$ for both concentrations).

\section{Discussion}

In a previous study, we demonstrated that a-PVA-SPION had no observable toxic effects on the survival of human immune cells; however, our analysis of cytokine secretion revealed that these particles trigger a dose-dependent release of certain cytokines in a manner which is comparable to 
an acute inflammatory process. ${ }^{25}$ It is difficult to compare results from different studies due to varying experimental conditions (ie, type of NP, NP concentration or incubation time, and cell types used); however the results of our previous study required further investigation. Therefore, the main goal of the present investigation was to identify the factors underlying our previous findings of increased cytokine production from blood cells after incubation with a-PVA-SPION, in order to generate a more suitable and safe theragnostic agent.

Our earlier study suggested that PVA is released from a-PVA-SPION; therefore, we hypothesized that free PVA may account, at least in part, for the observed effects of these NP on human immune cells. From in vivo studies, it is known that parenteral PVA administration can induce adverse effects. Experiments in rats demonstrated that PVA alone, administered intravenously at a dose of $460 \mathrm{mg} / \mathrm{kg}$ body weight, induced adverse hematological effects (increased neutrophils and decreased platelets), clinical chemistry indicative of liver effects (increased ALT and AST), and histopathology of the liver (hepatocellular necrosis). Such effects were either absent or less pronounced when the same dose of PVA was adsorbed on SPION. ${ }^{39}$

Our initial studies demonstrated that PVA does indeed induce a dose-dependent increase in IL1 $\beta$ secretion in human immune cells. Similar findings were recently reported in mice, ${ }^{40}$ where systemic administration of high-dose PVA provoked an inflammatory response. Gene expression of several pro-inflammatory proteins in the murine synovium and liver, as well as the serum cytokine concentrations of IL6 and keratinocyte chemoattractant, were increased. ${ }^{40}$

The aim of this study was to adapt the SPION, such that the release of PVA from the a-PVA-SPION complex was prevented. Therefore, we modified the PVA SPION coating to obtain covalently bonded a-PVA-SPION with the same physicochemical properties (size, charge, and surface chemistry) as the previously analyzed classical a-PVA-SPION. ${ }^{25}$ This was achieved by cross-linking with an amino-silica precursor, which also created a positive charge and amino chemical groups. The synthesis technique showed good reproducibility, high yield, and produced sufficient quantities per batch for experiments to study the biological effects of the formulation.

These newly engineered NP with PVA covalently bonded onto the SPION surface (a-PVA-grafted-SPION) were first analyzed to determine their effects on human cytokine secretion. In contrast to our expectations, cytokine secretion was still increased using this alternative NP preparation; however, the cytokines affected differed from those induced after incubation with a-PVA-SPION. ${ }^{25}$
The surface engineering of NP, which is crucial for their functionalization, plays an important role in their stability, biocompatibility, and half-life in blood..$^{26,41}$ Nevertheless, our results clearly demonstrate that other factors must also influence the effects of SPION on human immune cells, since the type of surface coating is similar in both formulations; although the PVA is not released from the complex in the a-PVA-grafted-SPION, cytokine secretion is still increased, albeit differently.

As the size and shape and the charge of NP are important in their interactions with cells, ${ }^{38}$ and the PVA cross-linking process led to a slight increase in the charge of a-PVAgrafted-SPION compared with a-PVA-SPION, we reduced the $\mathrm{Z}$ potential of a-PVA-grafted-SPION (++) further, and these adapted NP [a-PVA-grafted-SPION (+)] were analyzed for their interaction with human immune cells. This stepwise adaptation of the SPION to reduce the $\mathrm{Z}$ potential was successful, in that the resultant SPION had no negative influence on human immune cell survival and, compared with the increased cytokine secretion originally observed for the a-PVA-SPION, changes were reduced to a minimum. Only the chemokines MCP1 and MIP1b demonstrated increased secretion after exposure to a-PVA-grafted-SPION (+), with no increase in the levels of pro-inflammatory cytokines (eg, IL6, IL17). Whether the secretion of chemokines is advantageous, for example with regard to stimulation of immune cell migration into the inflamed tissue, remains to be clarified in future studies.

Finally, there is the question of why a slight reduction of $\mathrm{Z}$ potential (around $10 \mathrm{mV}$ ) caused such a large effect on $\mathrm{NP}-$ cell interaction. This may be due to the formation of a protein corona around the surface of the NP. It has been shown for polystyrene NP that the properties of NP determine the protein corona, with possible implications for biological impacts. ${ }^{42}$ This is consistent with a more recent study, in which PVA-SPION with varying $\mathrm{Z}$ potentials were incubated with fetal bovine serum at fixed serum/particle ratios. ${ }^{43}$ The analysis of tightly bound proteins revealed that neutral and positively charged PVA-coated NP bound a wide range of serum proteins, with similar patterns, whereas the number of bound proteins decreased on the surface of negatively charged PVA-NP. Interestingly, the number of bound protein types reduced around $40 \%$ in positively $(+13 \mathrm{mV})$, compared with highly positively $(+34 \mathrm{mV})$, charged particles. ${ }^{43}$ Other investigators have also demonstrated that protein corona depends on charge, and even conclude that this may lead to unintended uptake by the body (eg, the brain) with potentially cytotoxic effects of commercially available NP used for target-specific applications. ${ }^{44}$ 


\section{Conclusion and outlook}

In conclusion, we demonstrate that the surface characteristics of SPION strongly influence their biocompatibility. Our results prove that the newly formulated a-PVA-graftedSPION (+) are potentially suitable for use as a theragnostic agent, since they provoke fewer inflammatory effects, as a result of careful control and accurate definition of their surface chemistry. These factors should always be considered in future investigations of this kind.

Further developments of nanomaterials will facilitate the diagnosis and therapy of chronic inflammatory autoimmune diseases. NP are taken up by different cell types and provide a basis for targeted cell-based therapy. The various uptake mechanisms of NP are affected by their properties, including the type of surface coating and the surface charge. We clearly demonstrated that a small variation in the surface charge influences the effects of NP on cells of the immune system, which may, at least in part, be caused by the composition of the protein corona. Therefore, it may be advantageous to analyze protein coronas and the correlation of their composition with cellular uptake mechanisms. This could be beneficial for applications which aim to achieve site-directed targeting leading to very early treatment and/or visualization of tissue inflammation. This would enable early therapeutic intervention prior to the onset of significant joint damage in rheumatoid arthritis.

Future studies should focus on the composition of the protein corona and cellular uptake of the PVA-graftedSPION (+) developed in the present work.

\section{Acknowledgments}

We would like to thank Manuela Jakstadt for outstanding technical assistance, Heide Boeth for sample collection and archiving (both at Charité Berlin), and Marie-Gabrielle Beuzelin (EPFL Switzerland) and Usawadee Sakulkhu for their support.

\section{Disclosure}

This work was supported by the NanoDiaRA project, grant agreement number 228929, funded by the European Seventh Framework Programme FP7-NMP-2008-L.

The authors have no relevant affiliations or financial involvement with any organization or entity with a financial interest in, or financial conflict with, the subject matter or materials discussed in the manuscript, other than those disclosed.

\section{References}

1. Shubayev VI, Pisanic TR 2nd, Jin S. Magnetic nanoparticles for theragnostics. Adv Drug Deliv Rev. 2009;61(6):467-477.
2. Mahmoudi M, Sahraian MA, Shokrgozar MA, Laurent S. Superparamagnetic iron oxide nanoparticles: promises for diagnosis and treatment of multiple sclerosis. ACS Chem Neurosci. 2011;2(3):118-140.

3. Maier-Hauff K, Ulrich F, Nestler D, et al. Efficacy and safety of intratumoral thermotherapy using magnetic iron-oxide nanoparticles combined with external beam radiotherapy on patients with recurrent glioblastoma multiforme. J Neurooncol. 2011;103(2):317-324.

4. Laurent S, Saei AA, Behzadi S, Panahifar A, Mahmoudi M. Superparamagnetic iron oxide nanoparticles for delivery of therapeutic agents: opportunities and challenges. Expert Opin Drug Deliv. 2014; 11(9):1449-1470.

5. Jin R, Lin B, Li D, Ai H. Superparamagnetic iron oxide nanoparticles for MR imaging and therapy: design considerations and clinical applications. Curr Opin Pharmacol. 2014;18:18-27.

6. Heath JR. Nanotechnologies for biomedical science and translational medicine. Proc Natl Acad Sci U S A. 2015;112(47):14436-14443.

7. Mahmoudi M, Serpooshan V, Laurent S. Engineered nanoparticles for biomolecular imaging. Nanoscale. 2011;3(8):3007-3026.

8. Bulte JW, Kraitchman DL. Iron oxide MR contrast agents for molecular and cellular imaging. NMR Biomed. 2004;17(7):484-499.

9. Kosta PE, Voulgari PV, Zikou AK, Drosos AA, Argyropoulou MI. The usefulness of magnetic resonance imaging of the hand and wrist in very early rheumatoid arthritis. Arthritis Res Ther. 2011;13(3):R84.

10. Saraswathy A, Nazeer SS, Nimi N, Arumugam S, Shenoy SJ, Jayasree RS. Synthesis and characterization of dextran stabilized superparamagnetic iron oxide nanoparticles for in vivo MR imaging of liver fibrosis. Carbohydr Polym. 2014;101:760-768.

11. You DG, Saravanakumar G, Son S, et al. Dextran sulfate-coated superparamagnetic iron oxide nanoparticles as a contrast agent for atherosclerosis imaging. Carbohydr Polym. 2014;101:1225-1233.

12. Arruebo M, Fernández-Pacheco R, Ibarra MR, Santamaría J. Magnetic nanoparticles for drug delivery. Nano Today. 2007;2(3):22-32.

13. Mahmoudi M, Simchi A, Imani M, Häfeli UO. Superparamagnetic iron oxide nanoparticles with rigid cross-linked polyethylene glycol fumarate coating for application in imaging and drug delivery. The Journal of Physical Chemistry C. 2009;113(19):8124-8131.

14. Mahmoudi M, Hosseinkhani H, Hosseinkhani M, et al. Magnetic resonance imaging tracking of stem cells in vivo using iron oxide nanoparticles as a tool for the advancement of clinical regenerative medicine. Chem Rev. 2010;111(2):253-280.

15. Schulze F, Dienelt A, Geissler S, et al. Amino-polyvinyl alcohol coated superparamagnetic iron oxide nanoparticles are suitable for monitoring of human mesenchymal stromal cells in vivo. Small. 2014;10(21): 4340-4351.

16. Meyers JD, Doane T, Burda C, Basilion JP. Nanoparticles for imaging and treating brain cancer. Nanomedicine (Lond). 2013;8(1): 123-143.

17. Yen SK, Padmanabhan P, Selvan ST. Multifunctional iron oxide nanoparticles for diagnostics, therapy and macromolecule delivery. Theranostics. 2013;3(12):986-1003.

18. MacKenzie JD, Karasick D. Imaging of rheumatoid arthritis. In: Weissman BN, editor. Imaging of Arthritis and Metabolic Bone Disease. Elsevier Health Sciences; 2009:340-364.

19. Eckstein F, Burstein D, Link TM. Quantitative MRI of cartilage and bone: degenerative changes in osteoarthritis. NMR Biomed. 2006; 19(7):822-854.

20. Schett G. Erosive arthritis. Arthritis Res Ther. 2007;9 Suppl 1:S2.

21. Wang YX, Hussain SM, Krestin GP. Superparamagnetic iron oxide contrast agents: physicochemical characteristics and applications in MR imaging. Eur Radiol. 2001;11(11):2319-2331.

22. Gramoun A, Crowe LA, Maurizi L, et al. Monitoring the effects of dexamethasone treatment by MRI using in vivo iron oxide nanoparticlelabeled macrophages. Arthritis Res Ther. 2014;16(3):R131.

23. Lameijer MA, Tang J, Nahrendorf M, Beelen RH, Mulder WJ. Monocytes and macrophages as nanomedicinal targets for improved diagnosis and treatment of disease. Expert Rev Mol Diagn. 2013; 13(6):567-580. 
24. Lunov O, Zablotskii V, Syrovets T, et al. Modeling receptor-mediated endocytosis of polymer-functionalized iron oxide nanoparticles by human macrophages. Biomaterials. 2011;32(2):547-555.

25. Strehl C, Gaber T, Maurizi L, et al. Effects of PVA coated nanoparticles on human immune cells. Int J Nanomedicine. 2015;10:3429-3445.

26. Valdiglesias V, Kiliç G, Costa C, et al. Effects of iron oxide nanoparticles: cytotoxicity, genotoxicity, developmental toxicity, and neurotoxicity. Environ Mol Mutagen. 2015;56(2):125-148.

27. Jones CF, Grainger DW. In vitro assessments of nanomaterial toxicity. Adv Drug Deliv Rev. 2009;61(6):438-456.

28. Cromeans T, Humphrey C, Sobsey M, Fields H. Use of immunogold preembedding technique to detect hepatitis A viral antigen in infected cells. Am J Anat. 1989;185(2-3):314-320.

29. Raji MA, Amara M, Amoabediny G, et al. Cytotoxicity of synthesized iron oxide nanoparticles: toward novel biomarkers of colon cancer. Conf Proc IEEE Eng Med Biol Soc. 2014;2014:6179-6182.

30. Strehl C, Schellmann S, Maurizi L, et al. Effects of PVA-coated nanoparticles on human T helper cell activity. Toxicol Lett. 2016;245:52-58.

31. Maurizi L, Sakulkhu U, Crowe LA, et al. Syntheses of cross-linked polymeric superparamagnetic beads with tunable properties. RSC Adv. 2014;4(22):11142-11146.

32. Cornwell RM, Schwertmann U. The Iron Oxides: Structure, Properties, Reactions, Occurrence and Uses. 2nd ed. Weinheim: Wiley-VCH; 2006.

33. Chastellain M, Petri A, Gupta A, Rao KV, Hofmann H. Superparamagnetic silica-iron oxide nanocomposites for application in hyperthermia. Adv Eng Mater. 2004;6(4):235-241.

34. Maurizi L, Sakulkhu U, Gramoun A, Vallee JP, Hofmann H. A fast and reproducible method to quantify magnetic nanoparticle biodistribution. Analyst. 2014;139(5):1184-1191.

35. Jung CW, Jacobs P. Physical and chemical properties of superparamagnetic iron oxide MR contrast agents: ferumoxides, ferumoxtran, ferumoxsil. Magn Reson Imaging. 1995;13(5):661-674.
36. Jung CW. Surface properties of superparamagnetic iron oxide MR contrast agents: ferumoxides, ferumoxtran, ferumoxsil. Magn Reson Imaging. 1995;13(5):675-691.

37. Chastellain M, Petri A, Hofmann H. Particle size investigations of a multistep synthesis of PVA coated superparamagnetic nanoparticles. J Colloid Interface Sci. 2004;278(2):353-360.

38. Schweiger C, Hartmann R, Zhang F, Parak WJ, Kissel TH, Rivera Gil P. Quantification of the internalization patterns of superparamagnetic iron oxide nanoparticles with opposite charge. J Nanobiotechnology. 2012;10:28

39. Emde B, Weigt S, Kaufmann W, Johannes S. PVA-SPION-single dose intravenous study in rats plus a 14 day recovery period. Internal Study Report No T16240: Merck KGaA, Darmstadt, Germany; 2012.

40. Vermeij EA, Koenders MI, Bennink MB, et al. The in-vivo use of superparamagnetic iron oxide nanoparticles to detect inflammation elicits a cytokine response but does not aggravate experimental arthritis. PloS One. 2015;10(5):e0126687.

41. Kievit FM, Zhang M. Surface engineering of iron oxide nanoparticles for targeted cancer therapy. Acc Chem Res. 2011;44(10):853-862.

42. Lundqvist M, Stigler J, Elia G, Lynch I, Cedervall T, Dawson KA. Nanoparticle size and surface properties determine the protein corona with possible implications for biological impacts. Proc Natl Acad Sci US A. 2008;105(38):14265-14270.

43. Sakulkhu U, Mahmoudi M, Maurizi L, et al. Significance of surface charge and shell material of superparamagnetic iron oxide nanoparticle (SPION) based core/shell nanoparticles on the composition of the protein corona. Biomater Sci. 2015;3(2):265-278.

44. Mahmoudi M, Sheibani S, Milani AS, et al. Crucial role of the protein corona for the specific targeting of nanoparticles. Nanomedicine (Lond). 2015;10(2):215-226. 


\section{Supplementary materials}

A Step 1: washing

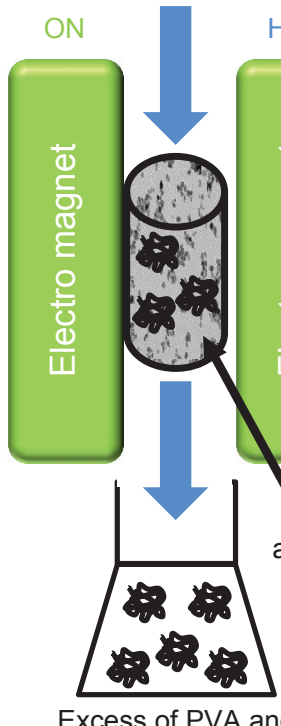

APTES
Step 2: release

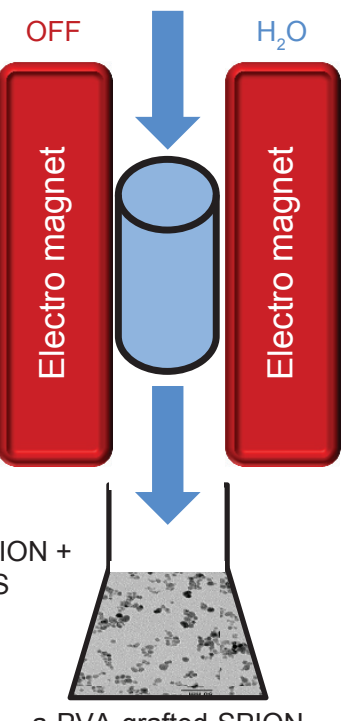

a-PVA-grafted-SPION
B

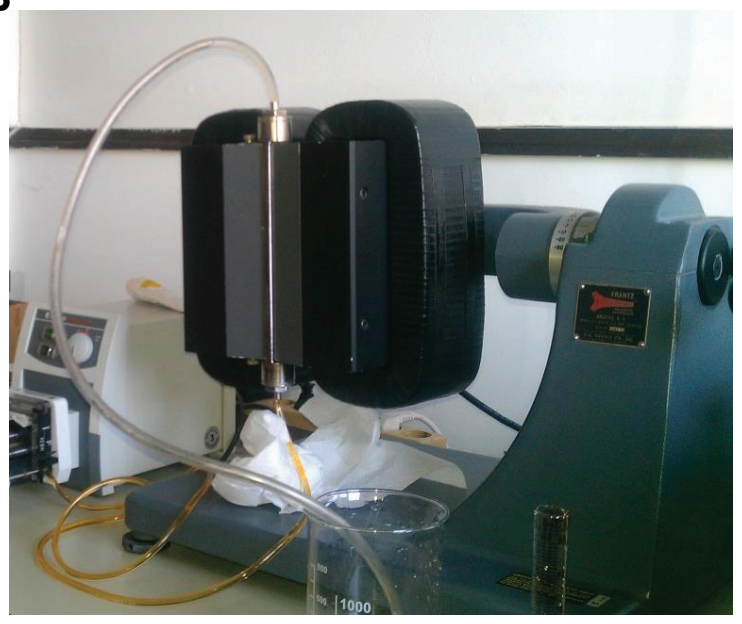

Figure SI Schematic representation (A) and photograph (B) of the magnetic reactor device used to obtain a-PVA-grafted-SPION.

Notes: In step I, the a-PVA-grafted-SPION were magnetically trapped for I hour and the excess of reactants washed out with DI water for 20 minutes. In step 2 the electromagnet was switched off and the washed a-PVA-grafted-SPION were released and stored.

Abbreviations: a-PVA, amino-polyvinyl alcohol; SPION, superparamagnetic iron oxide nanoparticles; DI, deionized; APTES, (3-aminopropyl)triethoxysilane; PVA, polyvinyl alcohol.
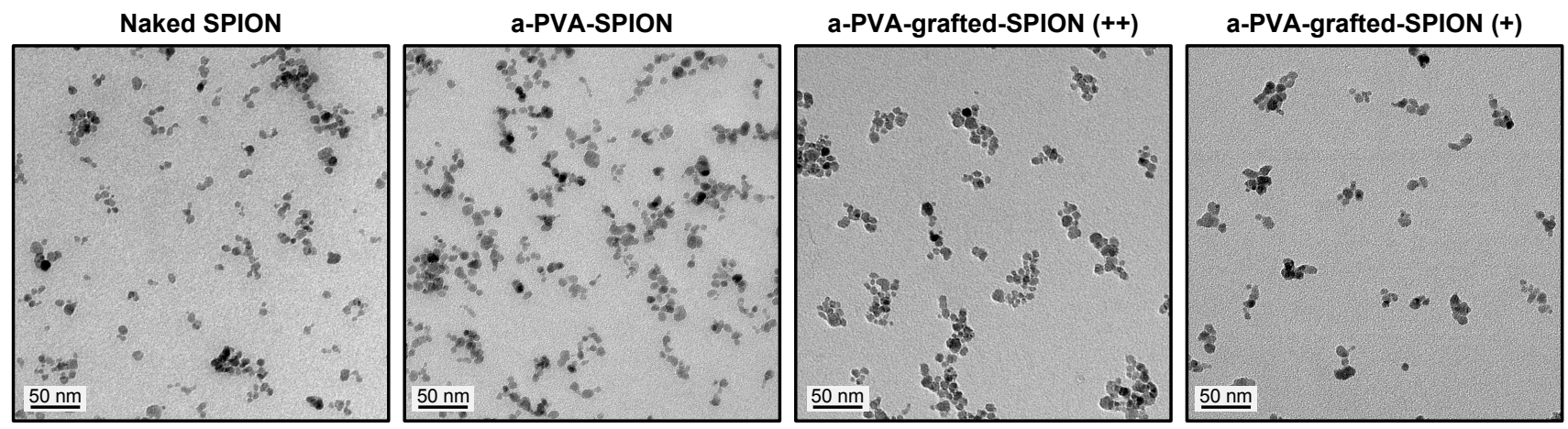

Figure S2 Transmission electron microscope (TEM) images.

Notes: Naked SPION and SPION coated with adsorbed amino-PVA (a-PVA-SPION), PVA grafted with amino cross-linker (a-PVA-grafted-SPION [++]), and PVA grafted with amino cross-linker and with decrease of the global positive charge (a-PVA-grafted-SPION [+]). It was not possible to visualize the PVA coating of SPION by TEM; however, their shape and sizes are not clearly altered, 45,000× magnification.

Abbreviations: SPION, superparamagnetic iron oxide nanoparticles; PVA, polyvinyl alcohol. 


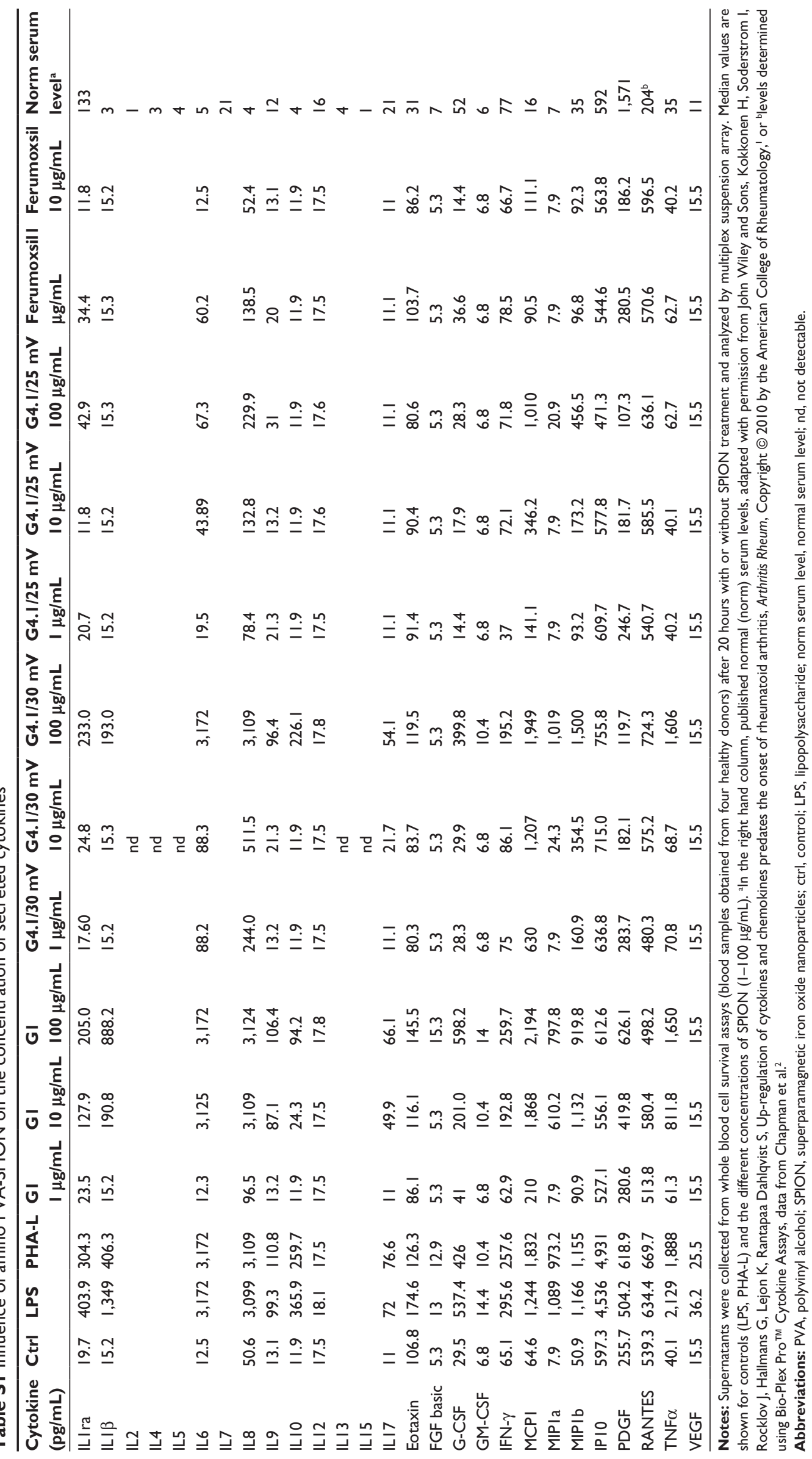




\section{References}

1. Kokkonen H, Soderstrom I, Rocklov J, Hallmans G, Lejon K, Rantapaa Dahlqvist S. Up-regulation of cytokines and chemokines predates the onset of rheumatoid arthritis. Arthritis Rheum. 2010;62(2):383-391.

2. Chapman P, Reyes C, Gupta V. Normal Physiological Levels of Human Cytokines Using Bio-Plex Pro ${ }^{\mathrm{TM}}$ Cytokine Assays. Bio-Rad Tech Note. 2010;6029. Available from: http://www.bio-rad.com/webroot/web/pdf/ 1sr/literature/Bulletin_6029.pdf. Accessed January 29, 2015.

\section{Publish your work in this journal}

The International Journal of Nanomedicine is an international, peerreviewed journal focusing on the application of nanotechnology in diagnostics, therapeutics, and drug delivery systems throughout the biomedical field. This journal is indexed on PubMed Central, MedLine, CAS, SciSearch ${ }^{\circledR}$, Current Contents ${ }^{\circledR} /$ Clinical Medicine,

Journal Citation Reports/Science Edition, EMBase, Scopus and the Elsevier Bibliographic databases. The manuscript management system is completely online and includes a very quick and fair peer-review system, which is all easy to use. Visit http://www.dovepress.com/ testimonials.php to read real quotes from published authors.

Submit your manuscript here: http://www.dovepress.com/international-journal-of-nanomedicine-journal 